\title{
Anomalous valley Hall effect in antiferromagnetic monolayers
}

\author{
Wenhui $\mathrm{Du}^{1}$, Rui Peng ${ }^{1}$, Zhonglin $\mathrm{He}^{1}$, Ying Dai ${ }^{1 凶}$, Baibiao Huang ${ }^{1}$ and Yandong Ma $\mathbb{\mathbb { D }}^{1 凶}$
}

Anomalous valley Hall (AVH) effect is a fundamental transport phenomenon in the field of condensed-matter physics. Usually, the research on $\mathrm{AVH}$ effect is mainly focused on $2 \mathrm{D}$ lattices with ferromagnetic order. Here, by means of model analysis, we present a general design principle for realizing AVH effect in antiferromagnetic monolayers, which involves the introduction of nonuniform potentials to break of $P T$ symmetry. Using first-principles calculations, we further demonstrate this design principle by stacking antiferromagnetic monolayer $\mathrm{MnPSe}_{3}$ on ferroelectric monolayer $\mathrm{Sc}_{2} \mathrm{CO}_{2}$ and achieve the AVH effect. The AVH effect can be well controlled by modulating the stacking pattern. In addition, by reversing the ferroelectric polarization of $\mathrm{Sc}_{2} \mathrm{CO}_{2}$ via electric field, the $\mathrm{AVH}$ effect in monolayer $\mathrm{MnPSe}_{3}$ can be readily switched on or off. The underlying physics are revealed in detail. Our findings open up a new direction of research on exploring AVH effect.

npj 2D Materials and Applications (2022)6:11 ; https://doi.org/10.1038/s41699-022-00289-6

\section{INTRODUCTION}

Valley, characterizing energy extrema of conduction or valence band, is an emerging degree of freedom of carriers in condensedmatter materials ${ }^{1,2}$. Benefiting from the large separation in momentum space, the valley degree of freedom is particularly stable against low-energy phonons and smooth deformations ${ }^{3-5}$. Analogous to charge and spin, the valley degree can be utilized to encode information and perform logic operations, leading to the concept of valleytronics ${ }^{6,7}$. Early interest in valley dates back to the works in 1970 s on silicon inversion layer ${ }^{8,9}$. In recent years, following the discoveries of intrinsic physical properties associated with valley occupancy in two-dimensional (2D) lattices ${ }^{10,11}$, rapid experimental and theoretical progress ${ }^{12-46}$ has been made in the field of valleytronics at both the fundamental and applied levels.

As a new piece of puzzle in the Hall family, the anomalous valley Hall (AVH) effect in valley-polarized materials lies at the heart of valleytronics. In principle, polarized light is able to induce AVH effect in valley-polarized monolayers, however, it is a dynamic process and subjected to the life time of carriers, which is not applicable for practical valleytronics ${ }^{27,28}$. Physically, there are two essential ingredients for realizing stable AVH effect: one is magnetism and the other is spin-orbital coupling (SOC), which, respectively, plays the role of breaking the time-reversal symmetry and giving rise to coupled spin and valley physics. The former can be either intrinsic magnetism ${ }^{29-36}$ or extrinsic magnetism caused by magnetic doping $^{37-40}$, magnetic field $\mathrm{d}^{41,42}$ and magnetic proximity effect ${ }^{43-46}$, while the latter is related to the chemical compositions. The past years have seen impressive progress for identifying such AVH effect in single-layer materials ${ }^{29-46}$. In all the prior studies, there is one default assumption, namely, the magnetism must be ferromagnetic. Compared with ferromagnetism, antiferromagnetic materials have great potential in the next generation of valleytronic and spintronic applications thanks to the intrinsic advantages such as robustness against magnetic field perturbation, ultrafast dynamics, stray fieldfree operation ${ }^{47,48}$. However, to our knowledge, it is still unclear how to realize stable AVH effect in single-layer materials with an antiferromagnetic order.

In the present work, we show by model analysis that the realization of stable $\mathrm{AVH}$ effect can be extended to single-layer materials with an antiferromagnetic order. The proposed general design principle for this extension correlates with the introduction of nonuniform potentials to break the $P T$ symmetry [invariance under the combined operation of spatial inversion $(P)$ and time reversal $(T)$ ]. Based on first-principles calculations, we further demonstrate this design principle by stacking antiferromagnetic monolayer $\mathrm{MnPSe}_{3}$ on ferroelectric monolayer $\mathrm{Sc}_{2} \mathrm{CO}_{2}$ and realize the AVH effect. Such AVH effect is shown to exhibit a tantalizing stacking pattern depended character. Moreover, we reveal that the AVH effect in monolayer $\mathrm{MnPSe}_{3}$ can be switched on or off by reversing the ferroelectric polarization of monolayer $\mathrm{Sc}_{2} \mathrm{CO}_{2}$. This extension of $\mathrm{AVH}$ effect to single-layer materials with an antiferromagnetic order is of great significance from both fundamental perspective and for potential use in devices.

\section{RESULTS AND DISCUSSION} Introducing nonuniform potentials to break PT symmetry Our proposed design principle for realizing AVH effect in singlelayer materials with an antiferromagnetic order is schematically illustrated in Fig. 1. Without losing the generality, we take monolayer $\mathrm{MnPSe}_{3}$ as an example to discuss the scheme by considering the fact that monolayer $\mathrm{MnPSe}_{3}$ is known as a typical Néel antiferromagnetic semiconductor with spontaneous valley polarization. Monolayer $\mathrm{MnPSe}_{3}$ exhibits a hexagonal lattice with the space group of $D_{3 d}$ (see Supplementary Fig. 1a). Without considering exchange interaction, its structure hosts the inversion symmetry $P$. In monolayer $\mathrm{MnPSe}_{3}$, the $\mathrm{Mn}$ atoms constitute two sublattices, which are referred to as $A$ and $B$ sublattices. Although the $A$ and $B$ sublattices are structurally equivalent, their spin orientations are opposite. In this regard, neither inversion symmetry $(P)$ nor time-reversal symmetry $(T)$ is preserved. However, it shows invariance under the simultaneous time reversal and spatial inversion, namely, PT symmetry. Because of the PT symmetry, the spin and valley polarization occur spontaneously in such antiferromagnetic monolayers, as schematically shown in Fig. 1a. Also protected by the PT symmetry, the valley spin splitting is prohibited, yielding the spin degeneracy for the $\mathrm{K}$ and $\mathrm{K}^{\prime}$ valleys. Such spin degeneracy in antiferromagnetic monolayers forbids the realization of AVH effect. For example, upon shifting Fermi level between the $\mathrm{K}$ and $\mathrm{K}^{\prime}$ valleys in the

\footnotetext{
${ }^{1}$ School of Physics, State Key Laboratory of Crystal Materials, Shandong University, Shandanan Str. 27, Jinan 250100, China. ${ }^{凶}$ email: daiy60@sina.com; yandong.ma@sdu.edu.cn
} 


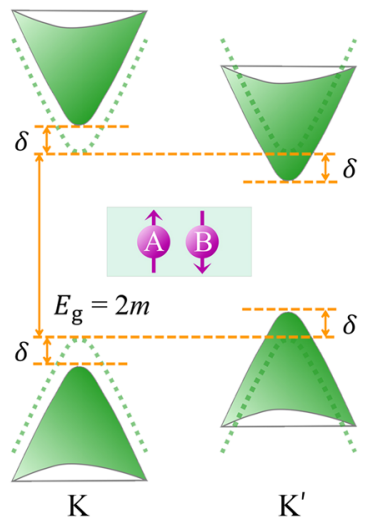

b

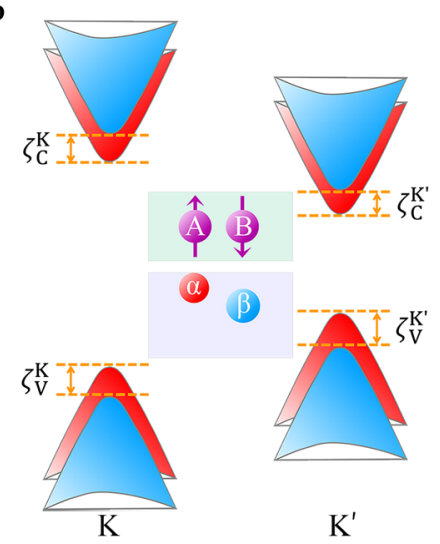

c

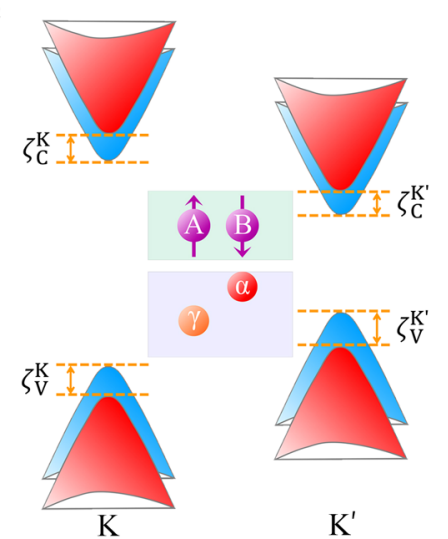

Fig. 1 Schematic band structures of monolayer $\mathrm{MnPSe}_{3}$. Schematic diagrams for the $\mathrm{K}$ and $\mathrm{K}^{\prime}$ valleys of monolayer $\mathrm{MnPSe}_{3}$ a without and $\mathbf{b}$, c with nonuniform potentials on the A and B sublattices. Dotted and solid cones in a represent the valleys without and with considering SOC, respectively. Red and blue cones in $\mathbf{b}$ and $\mathbf{c}$ correspond to two spin states, respectively.

conduction bands, the spin-up and spin-down electrons from the $\mathrm{K}^{\prime}$ valley would accumulate at opposite edges of the sample in the presence of an in-plane electric field, preventing the AVH effect.

If we introduce nonuniform potentials to neighboring $\mathrm{Mn}$ atoms, the equivalence of the A and B sublattices is deformed, which will break the PT symmetry. Without the protection of PT symmetry, the valley spin splitting at the $K$ and $K^{\prime}$ valleys would be achieved (see Fig. 1b), rendering the observation of AVH effect. Such valley spin splitting is different from the case in nonmagnetic materials, where the valley spin splitting relates to Zeeman splitting ${ }^{14,15,49}$. To preserve the valley physics, the nonuniform potentials introduced here should be regular. More importantly, it is natural to expect that the spin orders at both the $\mathrm{K}$ and $\mathrm{K}^{\prime}$ valleys can be reversed by reversing the nonuniform potentials introduced to A and B sublattices (Fig. 1C), which would enrich the AVH effect in antiferromagnetic monolayers.

Given the proposed design principle, we construct a k.p model to verify it. The spin-full Hamiltonian with excluding SOC for lowenergy quasiparticles near the $\mathrm{K}\left(\mathrm{K}^{\prime}\right)$ valley of a honeycomb lattice can be expressed as ${ }^{16}$

$H^{0}=v_{\mathrm{F}} s_{0}\left(\tau_{z} \sigma_{x} p_{x}+\tau_{0} \sigma_{y} p_{y}\right)+m s_{z} \tau_{0} \sigma_{z}$

Here, $p$ and $v_{\mathrm{F}}$ represent the momentum operator and the massless Fermi velocity, respectively. $\tau_{a}, \sigma_{a}$, and $s_{a}(a=x, y, z, 0)$ are the Pauli matrices for the valley, isospin, and spin degree of freedom, respectively. The mass term $(m)$, referring to an AFM perturbation, creates a band gap of $E_{\mathrm{g}}=2 \mathrm{~m}$ for both spins, preserving the spin and energy degeneracy. When taking SOC into account, the Hamiltonian for SOC is introduced as

$H^{\mathrm{SOC}}=\delta s_{z} \tau_{z} \sigma_{z}$

where $\delta$ is the SOC parameter. In the presence of SOC, the band gap is enlarged by $2 \delta$ at the $\mathrm{K}$ valley and reduced by $2 \delta$ at the $\mathrm{K}^{\prime}$ valley. As a result, the energetic degeneracy of the $K$ and $K^{\prime}$ valleys are lifted, but the spin degeneracy is preserved due to the invariance of the $H^{0}+H^{\text {SOC }}$ under simultaneous time reversal and spatial inversion. The term for the nonuniform potential between two sublattices can be written as:

$H_{\mathrm{ex}}=\frac{\zeta}{2} s_{0} \tau_{0} \sigma_{z}$

where $\frac{\zeta}{2}$ represents the strength of the nonuniform potential. In this case, the $H^{0}+H^{\mathrm{SOC}}+H^{\mathrm{ex}}$ would not be invariance under simultaneous time reversal and spatial inversion. This drives the bands from one sublattice to shift upward with respect to those from the other, resulting in a valley spin splitting of $\zeta$ at the $K$ and $\mathrm{K}^{\prime}$ valleys. According to the total Hamiltonian $H=H^{0}+H^{\mathrm{SOC}}+H^{\mathrm{ex}}$, the eigenvalues of the highest valence bands are estimated to be $\left(-m-\delta-\frac{\zeta}{2}\right)$ and $\left(-m-\delta+\frac{\zeta}{2}\right)$ for spin-up and spin-down at the $\mathrm{K}$ valley, respectively, and $\left(-m+\delta-\frac{\zeta}{2}\right)$ and $\left(-m+\delta+\frac{\zeta}{2}\right)$ for spin-up and spin-down at $\mathrm{K}^{\prime}$ valley. And the eigenvalues of the lowest conduction bands are found to be $\left(m+\delta+\frac{\zeta}{2}\right)$ and $\left(m+\delta-\frac{\zeta}{2}\right)$ for spin-up and spindown at the $\mathrm{K}$ valley, respectively, and $\left(m-\delta+\frac{\zeta}{2}\right)$ and $\left(m-\delta-\frac{\zeta}{2}\right)$ for spin-up and spin-down at $K^{\prime}$ valley. When reversing the nonuniform potential between two sublattices, the sign of $H_{\mathrm{ex}}$ is reversed, leading to the opposite valley spin splitting at the $K$ and $\mathrm{K}^{\prime}$ valleys. These results firmly indicate that the proposed design principle for realizing $\mathrm{AVH}$ effect in antiferromagnetic monolayers is feasible physically.

\section{Stacking-dependent AVH effect in $\mathrm{MnPSe}{ }_{3} / \mathrm{Sc}_{2} \mathrm{CO}_{2}$}

Having established the feasibility of the proposed design principle, next we discuss its realization in real materials. The proximity effect is proposed here to introduce nonuniform potentials on the $A$ and $B$ sites regularly. For detail, we consider monolayer $\mathrm{Sc}_{2} \mathrm{CO}_{2}$ as the substrate to induce the proximity effect in monolayer $\mathrm{MnPSe}_{3}$. The crystal structure of monolayer $\mathrm{Sc}_{2} \mathrm{CO}_{2}$ is shown in Supplementary Fig. 1b. It exhibits a hexagonal lattice with the space group P3m1. Due to the asymmetric displacement of inner $\mathrm{C}$ atomic layer with respect to Sc atomic layers, it hosts intrinsic ferroelectricity with out-of-plane polarization ${ }^{50}$. The band structure shown in Supplementary Fig. 1d suggests that monolayer $\mathrm{Sc}_{2} \mathrm{CO}_{2}$ is a semiconductor with an indirect band gap of $1.79 \mathrm{eV}$. Concerning the stacking between monolayer $\mathrm{MnPSe}_{3}$ and $\mathrm{SC}_{2} \mathrm{CO}_{2}$, a $2 \times 2$ supercell of $\mathrm{SC}_{2} \mathrm{CO}_{2}$ is adopted to match the unit cell of $\mathrm{MnPSe}_{3}$. This results in a compressive strain of about $6 \%$ for $\mathrm{Sc}_{2} \mathrm{CO}_{2}$ layer, which is in an accessible range in experiments ${ }^{51,52}$. It should be noted that when two van der Waals (vdW) materials are brought into contact, the lattice would undergo relaxation, while could result in lattice reconstructions as well as strain ${ }^{53}$. To reveal strain effect on AVH physics, we also investigate the band structures of $\mathrm{MnPSe} \mathrm{S}_{3} / \mathrm{SC}_{2} \mathrm{CO}_{2}$ under different tensile strain, and similar results are obtained (see Supplementary Fig. 2). According to the previous works ${ }^{46,54-56}$, three typical stacking patterns (i.e., h- $I_{\downarrow}, \mathrm{h}-\mathrm{Il}_{\downarrow}$ and $\mathrm{h}-\mathrm{II} \mathrm{l}_{\downarrow}$, as shown in Fig. 2a) with the polarization of $\mathrm{Sc}_{2} \mathrm{CO}_{2}$ pointing away from the interface are considered for the stacking between monolayer $\mathrm{MnPSe}_{3}$ and $\mathrm{Sc}_{2} \mathrm{CO}_{2}$. These three patterns can induce three distinct proximity effects on $\mathrm{MnPSe}_{3}$. In $\mathrm{h}-\mathrm{I}_{\downarrow}$ pattern, the $\mathrm{Mn}_{1}$ atoms sit above the top-O sites of $\mathrm{Sc}_{2} \mathrm{CO}_{2}$, and the $\mathrm{Mn}_{2}$ atoms are right above the $\mathrm{Sc}$ atoms in the second layer of $\mathrm{Sc}_{2} \mathrm{CO}_{2}$. In h-II atoms lie above the $C$ atoms in the middle layer, and the $M_{2}$ atoms are right above the top-O atoms. In h-III $\downarrow$ pattern, the $\mathrm{Mn}_{1}$ atoms sit above the $\mathrm{Sc}$ atoms in the second layer, and $\mathrm{Mn}_{2}$ atoms 


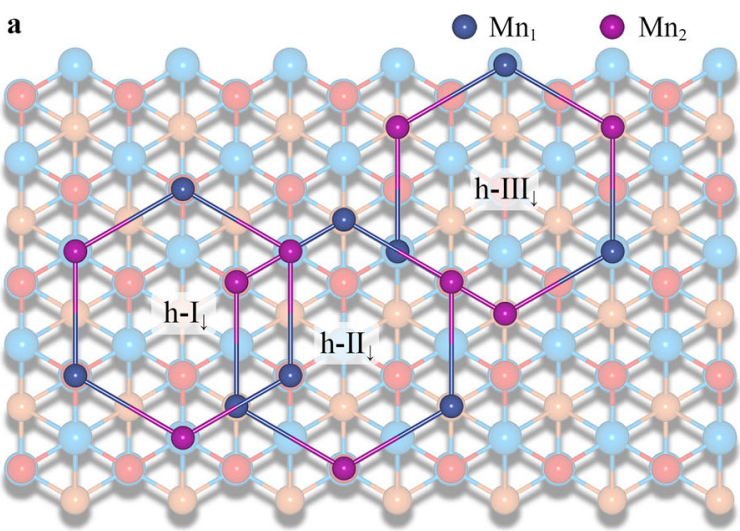

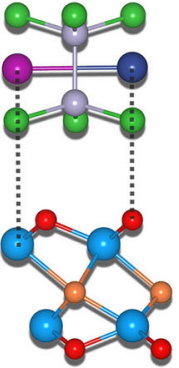

h-I $\mathrm{I}_{\downarrow}$

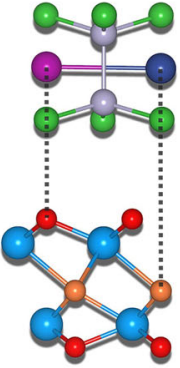

h-II

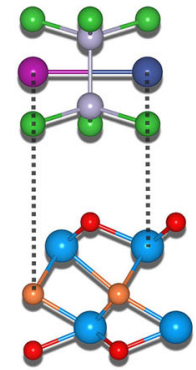

h-III
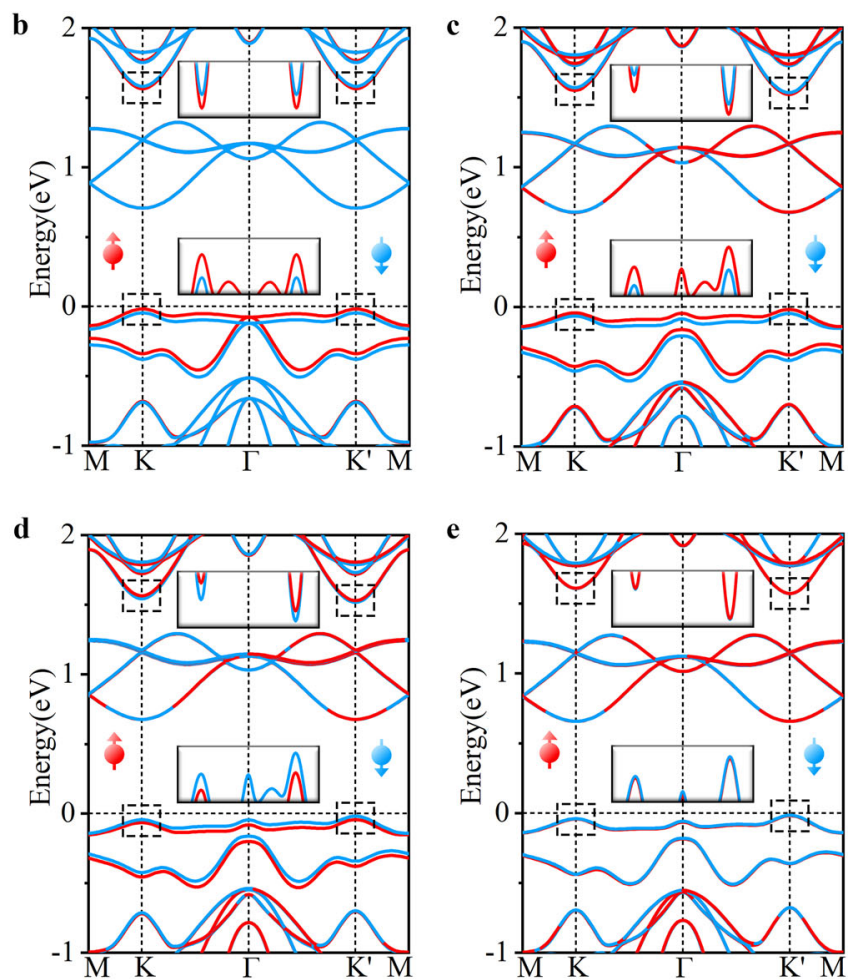

Fig. 2 Structural configurations and band structures. a Crystal structures of $\mathrm{h}-\mathrm{I}_{\downarrow}$, $\mathrm{h}-\mathrm{II}, \mathrm{and} \mathrm{h}-\mathrm{III}$. Band structures of h-I $\mathbf{b}$ without and $\mathbf{c}$ with considering SOC. $\mathbf{d}$ Band structure of $\mathrm{h}-\mathrm{Il}_{\downarrow}$ with considering SOC. e Band structure of h-III $\downarrow$ with considering SOC. Blue and red lines in $\mathbf{b}-\mathbf{e}$ correspond to spin-down and spin-up states, respectively. The Fermi level is set to $0 \mathrm{eV}$.

lie above the $\mathrm{C}$ atom in the middle layer of $\mathrm{Sc}_{2} \mathrm{CO}_{2}$. Concerning other stacking patterns, the proximity effect is expected to be similar to or in between the cases of $\mathrm{h}-\mathrm{I}_{\downarrow} / \mathrm{II}_{\downarrow} / \mathrm{III} \mathrm{I}_{\downarrow}$ configurations. Taking the configuration with $\mathrm{Mn}$ atom sitting above the center of Sc-O-C triangle as an example, we calculate its band structure. As shown in Supplementary Fig. 3, the scenario is similar to that of $\mathrm{h}-\mathrm{III} \downarrow$ configuration.

In $\mathrm{h}-\mathrm{I}_{\downarrow}$ configuration, the $\mathrm{Mn}_{1}$ atoms would experience an extra interaction due to their proximity to the top-O sites of $\mathrm{Sc}_{2} \mathrm{CO}_{2}$, while the $\mathrm{Mn}_{2}$ atoms would endure with a relatively small interaction as they lie farther. This can be described by the scenario illustrated in Fig. $1 \mathrm{~b}$, which introduces nonuniform potentials into the two sublattices of $\mathrm{Mn}$ atoms. The band structure of $\mathrm{h}-\mathrm{I}$ configuration without considering SOC is shown in Fig. $2 \mathrm{~b}$ and Supplementary Fig. 4a. The conduction band minimum (CBM) and valence band maximum (VBM) are contributed by monolayer $\mathrm{Sc}_{2} \mathrm{CO}_{2}$ and $\mathrm{MnPSe}_{3}$, respectively, forming a type-II band alignment. The degenerate valleys of $\mathrm{MnPSe}_{3}$ in both the conduction and valence bands locating at the $\mathrm{K}$ and $\mathrm{K}^{\prime}$ points are preserved after stacking on $\mathrm{SC}_{2} \mathrm{CO}_{2}$. However, as shown in Fig. $2 b$, the interesting valley spin splitting occurs in both the bottom conduction and top valence bands of $\mathrm{MnPSe}_{3}$ at the $\mathrm{K} / \mathrm{K}^{\prime}$ point, which correlates to the nonuniform potentials induced by stacking. Although the valleys of $\mathrm{MnPSe}_{3}$ in the conduction bands submerge in the bands of $\mathrm{SC}_{2} \mathrm{CO}_{2}$, they are still investigated for comparison. The valley spin splitting in the valence bands ( $\left.\zeta_{V}^{K}=\zeta_{V}^{K^{\prime}}\right)$ and conduction bands $\left(\zeta_{C}^{K}=\zeta_{C}^{K^{\prime}}\right)$ are estimated to be 28.5 and $16.7 \mathrm{meV}$, respectively. Such sizeable valley spin splitting stands in sharp contrast to the valley spin splitting in nonmagnetic materials which are induced by SOC and $P$ breaking.

When taking SOC into account, as shown in Fig. 2c, the character of valley spin splitting of $\mathrm{MnPSe}_{3}$ is maintained at both the $K$ and $K^{\prime}$ valleys, but the values of valley spin splitting at $K$ and $\mathrm{K}^{\prime}$ valleys are no longer equal; see Supplementary Table 1 . In this case, the energetic degeneracy of the $\mathrm{K}$ and $\mathrm{K}^{\prime}$ valleys in $\mathrm{MnPSe}_{3}$ is lifted, leading to the spontaneous valley polarization. Particularly, the valley polarization in the valence band is as large as $24.6 \mathrm{meV}$. The coexistence of valley spin splitting and valley polarization would ensure the observation of AVH effect in antiferromagnetic monolayer of $\mathrm{MnPSe}_{3}$, as we will show later.

As discussed above, the valley spin splitting relates to the nonuniform potentials induced by the proximity effect. Therefore, by engineering the stacking pattern, the valley spin splitting can be modulated. In $\mathrm{h}-I_{\downarrow}$ configuration, the $\mathrm{Mn}_{2}$ atoms experience an extra interaction due to their proximity to the top-O sites of $\mathrm{Sc}_{2} \mathrm{CO}_{2}$, while the $\mathrm{Mn}_{1}$ atoms endure with a relatively small interaction as they lie farther. This corresponds to the scenario illustrated in Fig. 1c. Accordingly, the nonuniform potentials introduced to $M n_{1}$ and $M n_{2}$ atoms in $h-I_{\downarrow}$ configuration is reversed with respect to those in $h-I_{\downarrow}$ configuration. The band structure of $h-I_{\downarrow}$ configuration with considering SOC is presented in Fig. $2 d$. It shares similar features to the band structure of $h-I_{\downarrow}$. The interesting valley spin splitting and spontaneous valley polarization are observed at the $\mathrm{K}$ and $\mathrm{K}^{\prime}$ valleys of $\mathrm{MnPSe}_{3}$, and the magnitudes are comparable to that in $h-I_{\downarrow}$ configurations. However, the sign of the valley spin splitting is opposite to that of $h-I_{\downarrow}$ configurations, which is consistent with the proposed design principle. Such simultaneous existence of valley spin splitting and valley polarization would facilitate the $\mathrm{AVH}$ effect as well.

Different from the cases of $h-I_{\downarrow}$ and $h-I_{\downarrow}$ configurations, both the $M n_{1}$ and $M n_{2}$ atoms in $h-I I I_{\downarrow}$ configuration experience a weak extra interaction since they lie far away from the atoms of $\mathrm{Sc}_{2} \mathrm{CO}_{2}$. Thus, the nonuniform potentials introduced to $M_{1}$ and $M_{2}$ atoms by the proximity effect would be rather weak. According to the proposed design principle, the valley spin splitting of $\mathrm{MnPSe}_{3}$ in $\mathrm{h}-\mathrm{III}_{\downarrow}$ configuration would be tiny. The band structure of $\mathrm{h}-\mathrm{III}_{\downarrow}$ configuration with considering SOC is displayed in Fig. 2e. As expected, the valley spin splitting is as small as $2.3 \mathrm{meV}(2.6 \mathrm{meV})$ at the $K\left(K^{\prime}\right)$ valley in the valence band, while it is even $1 \mathrm{meV}$ ( $1 \mathrm{meV}$ ) in the conduction band of $\mathrm{MnPSe}_{3}$, although large 
spontaneous valley polarization is preserved. In this case, the observation of $\mathrm{AVH}$ effect in $\mathrm{h}-\mathrm{III}_{\downarrow}$ configuration is difficult to achieve. Therefore, the valley spin splitting in $\mathrm{MnPSe}_{3}$ is indeed stacking dependent and thus can be engineered by modulating the stacking pattern, while holds great promise for practical applications. There are two points we wish to stress. First, currently, the stacking orders of $2 \mathrm{D}$ vdW materials can be well modulated in experiments ${ }^{57,58}$. Second, although the three stacking patterns might coexist in macroscopic area, the different configurations would be well-separated and the domains for them would be well-defined ${ }^{59,60}$.

The nonuniform potentials introduced to $M n_{1}$ and $M n_{2}$ atoms by the proximity effect for all these three stacking patterns can also be straightforwardly reflected by the variation of magnetic moments. For freestanding $\mathrm{MnPSe}_{3}$, the magnetic moments on $M n_{1}$ and $M n_{2}$ atoms are found to be 4.52 and $-4.52 \mu_{B}$, respectively, which is protected by the $P T$ symmetry. When stacking $\mathrm{MnPSe}_{3}$ on $\mathrm{Sc}_{2} \mathrm{CO}_{2}$ to form the $\mathrm{h}-\mathrm{I}_{\downarrow}$ configuration, the magnetic moment on $\mathrm{Mn}_{1}$ atom changes to $4.508 \mu_{\mathrm{B}}$, while the magnetic moment on $\mathrm{Mn}_{2}$ atom experiences with a relatively slight change $\left(-4.513 \mu_{\mathrm{B}}\right)$. This suggests the nonuniform potentials on the $M n_{1}$ and $M n_{2}$ atoms. As compared with h-I $\downarrow$ configuration, the absolute values of the magnetic moments on $M n_{1}$ and $M_{2}$ atoms in $\mathrm{h}-I_{\downarrow}$ configuration are exchanged, but the signs of the magnetic moments remain the same. Namely, the magnetic moments on $\mathrm{Mn}_{1}$ and $\mathrm{Mn}_{2}$ atoms in $\mathrm{h}-\mathrm{Il}_{\downarrow}$ configuration are found to be 4.513 and $-4.508 \mu_{\mathrm{B}}$, respectively. This can be easily understood by recalling the reversal of the atomic environments for $\mathrm{Mn}_{1}$ and $M n_{2}$ atoms in $\mathrm{h}-\mathrm{I}_{\downarrow}$ and $\mathrm{h}-\mathrm{II}_{\downarrow}$ configurations. For $\mathrm{h}-\mathrm{III}_{\downarrow}$ configuration, the magnetic moments on $\mathrm{Mn}_{1}$ and $\mathrm{Mn}_{2}$ atoms slightly change to 4.514 and $-4.514 \mu_{\mathrm{B}}$, respectively. The preservation of identical absolute values of the magnetic moments on $\mathrm{Mn}_{1}$ and $\mathrm{Mn}_{2}$ atoms in $\mathrm{h}-\mathrm{III}_{\downarrow}$ configuration relates to the weak extra interactions on both $\mathrm{Mn}$ atoms.

In 2D hexagonal lattices with inversion symmetry breaking, the $\mathrm{K}$ and $\mathrm{K}^{\prime}$ valleys will exhibit a nonzero Berry curvature along the out-of-plane direction. The Berry curvature is defined as ${ }^{61}$

$\Omega(\mathbf{k})=-\sum_{n} \sum_{n \neq n^{\prime}} f_{n} \frac{2 \operatorname{lm}\left\langle\psi_{n \mathbf{k}}\left|v_{x}\right| \psi_{n^{\prime} \mathbf{k}}\right\rangle\left\langle\psi_{n^{\prime} \mathbf{k}}\left|v_{y}\right| \psi_{n \mathbf{k}}\right\rangle}{\left(E_{n}-E_{n^{\prime}}\right)^{2}}$

Here, $f_{n}$ is the Fermi-Dirac distribution function, $\psi_{n \mathbf{k}}$ is the Bloch wave function with eigenvalue $E_{n}$, and $v_{x} / v_{y}$ is the velocity operator along $x / y$ direction. The calculated Berry curvatures of the spin-up and spin-down channels over the 2D Brillouin zone for monolayer $\mathrm{MnPSe}_{3}$ are shown in Fig. 3a. Obviously, the Berry curvatures for the same spin state around the $K$ and $K^{\prime}$ valleys are opposite, and the Berry curvatures for spin-up and spin-down states at the same valley are opposite as well. Note that under a longitudinal in-plane electric field, the Bloch carriers will acquire an anomalous transverse velocity proportional to the Berry curvature: $v_{\mathrm{a}} \sim E \times \Omega(\mathbf{k})$. In monolayer $\mathrm{MnPSe}_{3}$, when shifting the Fermi level between the $\mathrm{K}$ and $\mathrm{K}^{\prime}$ valleys in the valence band, protected by the $P T$ symmetry, the spin-down and spin-up holes from the $K^{\prime}$ valley will gather to opposite edges of the sample in the presence of an in-plane electric field, see Fig. $3 \mathrm{~b}$. In this regard, a net spin current is generated, which results in the spin Hall effect, however, the AVH effect is absent. In h- $\left.\right|_{\downarrow}$ configuration, because of the additional valley spin splitting, the spin degeneracy is lifted. When shifting the Fermi level between the $K$ and $K^{\prime}$ valleys in the valence band, only the spin-down holes from the $K^{\prime}$ valley move to the bottom boundary of the sample under an inplane electric field; see Fig. 3c. This gives rise to the long sought AVH effect in antiferromagnetic monolayers. In addition, the accumulated spin-down holes will result in a net charge/spin current. Similar to the case of $h-I_{\downarrow}$ configuration, when shifting the Fermi level between the $K$ and $\mathrm{K}^{\prime}$ valleys in the valence band of the $\mathrm{h}-\mathrm{Il}_{\downarrow}$ configuration, the spin-up holes from the $\mathrm{K}^{\prime}$ valley will accumulate at the top edge of the sample (Fig. 3d), which results into the AVH effect as well.

\section{Ferroelectrically controllable AVH effect}

Note that monolayer $\mathrm{Sc}_{2} \mathrm{CO}_{2}$ is a $2 \mathrm{D}$ ferroelectric crystal with an outof-plane polarization, its polarization direction can be reversed under a short-term out-of-plane electric field. Note that the reversed polarized state will be retained when the external electric field is removed. Such ferroelectric switching might affect the electronic properties of the $\mathrm{MnPSe}_{3} / \mathrm{Sc}_{2} \mathrm{CO}_{2}$. In the following, we investigate the properties of the three typical stacking patterns with switching the ferroelectric polarization of $\mathrm{Sc}_{2} \mathrm{CO}_{2}$ from downward to upward, which are referred to as $\mathrm{h}-\mathrm{I}_{\uparrow}, \mathrm{h}-\mathrm{II}_{\uparrow}, \mathrm{h}-\mathrm{III}_{\uparrow}$, respectively; see Supplementary Fig. 5. Figure $4 a$ presents the band structures of $\mathrm{h}-\mathrm{I}_{\uparrow}, \mathrm{h}-\mathrm{II}_{\uparrow}$ and $\mathrm{h}-\mathrm{III}_{\uparrow}$ configurations with considering SOC. The band structures of all these three configurations share similar characters. In all these three configurations, the Fermi level crosses the bottom of the conduction band and top of the valence band, yielding a metallic nature. More interestingly, the valleys of $\mathrm{MnPSe}_{3}$ in both the conduction and valence bands are submerged in the trivial bands of $\mathrm{Sc}_{2} \mathrm{CO}_{2}$, restricting any potential utilization of these valleys. In other word, under the ferroelectric switching, all these three configurations undergo a semiconductor-to-metal transition. Such transition is also accompanied with the disappearance of valley physics as well as the AVH effect. Accordingly, the AVH effect in $h-I_{\downarrow}$ and $h-I_{\downarrow}$ configurations is ferroelectrically controllable, benefiting for developing controllable valleytronic devices.

To understand the underlying mechanism of the ferroelectricpolarization-dependent properties, we calculate the planeaverage electrostatic potentials of monolayer $\mathrm{MnPSe}_{3}$, monolayer $\mathrm{Sc}_{2} \mathrm{CO}_{2}, \mathrm{~h}-\mathrm{I}_{\downarrow}$ and $\mathrm{h}-\mathrm{I}_{\uparrow}$ (Fig. $4 \mathrm{~b}$ and Supplementary Fig. 6). It can be seen that arising from the asymmetric structure, intrinsic polarization occurs along the out-of-plane direction, leading to the different work functions at the two sides of monolayer $\mathrm{SC}_{2} \mathrm{CO}_{2}$. Accordingly, upon contacting monolayer $\mathrm{MnPSe}_{3}$ with $\mathrm{Sc}_{2} \mathrm{CO}_{2}$ in different polarized states, the distinctly different band alignments forms, as shown in Fig. 4c. When the polarization of monolayer $\mathrm{Sc}_{2} \mathrm{CO}_{2}$ points away from the interface, the $\mathrm{CBM}$ of $\mathrm{Sc}_{2} \mathrm{CO}_{2}$ locates above the VBM of $\mathrm{MnPSe}_{3}$, inhibiting the transfer of electrons between $\mathrm{Sc}_{2} \mathrm{CO}_{2}$ and $\mathrm{MnPSe}_{3}$. The valley feature in the valence band from $\mathrm{MnPSe}_{3}$ is preserved. In contrast, when the polarization pointing to the interface, the CBM of $\mathrm{MnPSe}_{3}$ shifts below the VBM of $\mathrm{SC}_{2} \mathrm{CO}_{2}$, prompting the electron transfer from $\mathrm{Sc}_{2} \mathrm{CO}_{2}$ to $\mathrm{MnPSe}_{3}$, leading to the metallic nature. By comparing Supplementary Figs. 1c, $d$ and 4, it can be seen that the bands from the constituent layers are roughly similar to the corresponding freestanding ones, which indicates the weak hybridization between them for both the upward-polarization and down-polarization cases. The polarization reversal only changes the band alignment and gives rise to different band contributions around the Fermi level, leading to the submersion of valleys in the trivial bands or not. The charge transfer character between $\mathrm{Sc}_{2} \mathrm{CO}_{2}$ to $\mathrm{MnPSe}_{3}$ in $\mathrm{h}-\mathrm{I}_{\uparrow}, \mathrm{h}-\mathrm{II}_{\uparrow}$ and $\mathrm{h}-\mathrm{III}_{\uparrow}$ configurations is also confirmed by the charge density differences shown in Supplementary Fig. 7. While for $h-I_{\downarrow}$, $\mathrm{h}-\mathrm{Il}_{\downarrow}$ and $\mathrm{h}-\mathrm{III}_{\downarrow}$ configurations, the charge redistribution occurs around the interface. The charge transfer caused by polarization reversal could lead to semiconductor-to-metal transition. Such transition is accompanied with the submersion of valleys in the trivial bands and thus deforms the valley physics. While for the case under hole doping, it will not lead to the submersion of valleys in the trivial bands, which could preserve the valley physics. With these results in hand, we can understand the polarization dependent behaviors in $\mathrm{MnPSe} / \mathrm{Sc}_{2} \mathrm{CO}_{2}$.

To summarize, through model analysis, a general design principle for realizing stable $\mathrm{AVH}$ effect in antiferromagnetic monolayers is proposed, which involves the introduction of nonuniform potentials to $M n_{1}$ and $M n_{2}$ atoms to break the $P T$ symmetry. Using 


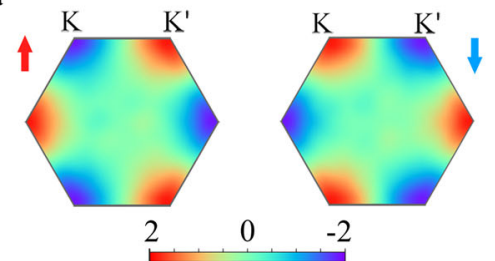

c

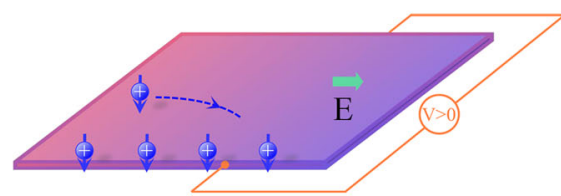

b

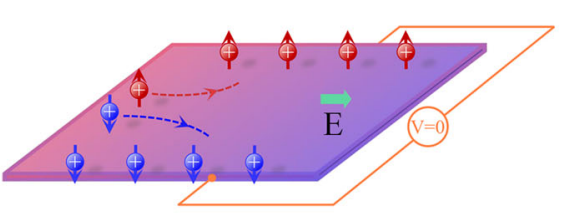

d

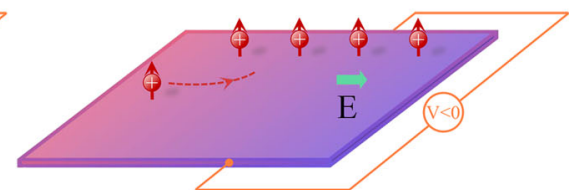

Fig. 3 Berry curvatures and AVH effect. a Berry curvatures of the spin-up and spin-down channels over the 2D Brillouin zone for monolayer $\mathrm{MnPSe}_{3}$. b Diagram of the spin and valley Hall effects in monolayer MnPSe ${ }_{3}$ under hole doping in presence of an in-plane electric field. Diagrams of the spin Hall and AVH effects in $\mathbf{c} h-I_{\downarrow}$ and $\mathbf{d} \mathbf{h}-I_{\downarrow}$ configurations under hole doping in presence of an in-plane electric field. Red/ blue arrows in $\mathbf{b}-\mathbf{d}$ indicate spin-up/down states, and the " $+" l^{\prime \prime}-"$ "symbols indicate holes/electrons from the $\mathrm{K}^{\prime}$ valley.

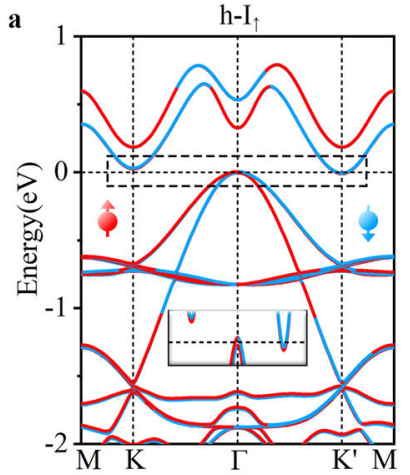

b

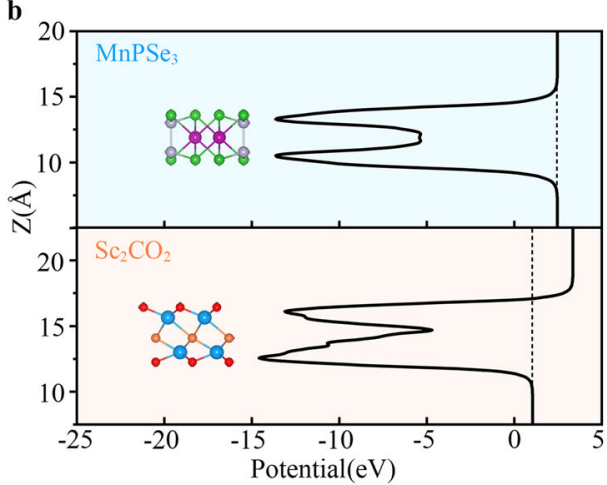

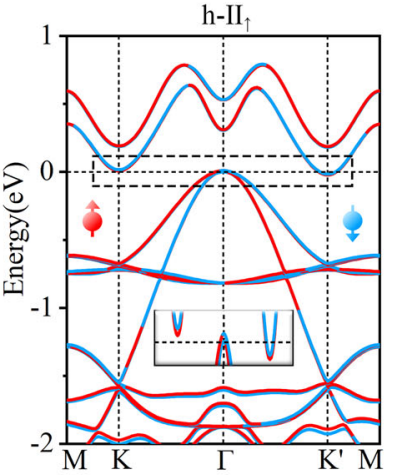
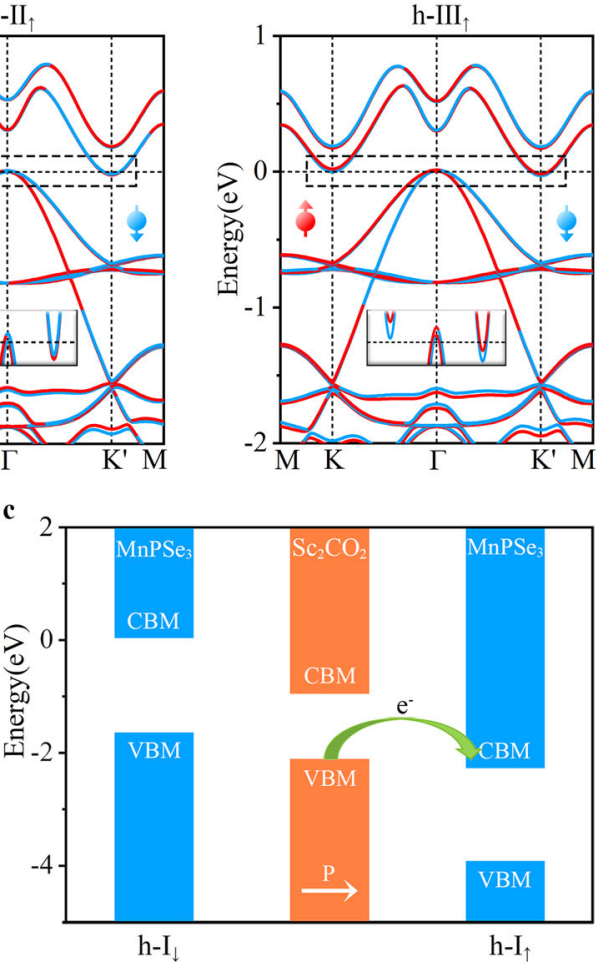

Fig. 4 Ferroelectric-polarization-dependent properties in $\mathrm{MnPSe}_{3} / \mathrm{Sc}_{2} \mathrm{CO}_{2}$. Band structures of $\mathrm{h}-\mathrm{I}_{\uparrow}$, h-II and $\mathrm{h}-\mathrm{III}_{\uparrow}$ with considering SOC. Blue and red lines in a correspond to spin-down and spin-up states, respectively. The Fermi level is set to $0 \mathrm{eV}$. $\mathbf{b}$ Plane-averaged electrostatic potentials of monolayer $\mathrm{MnPSe}_{3}$ and $\mathrm{Sc}_{2} \mathrm{CO}_{2}$ along the $z$ direction. c Band alignments of $h-I_{\downarrow}$ and $h-I_{\uparrow}$ with respect to the vacuum level.

first-principles calculations, the proposed design principle is further demonstrated by stacking antiferromagnetic monolayer $\mathrm{MnPSe}_{3}$ on ferroelectric monolayer $\mathrm{Sc}_{2} \mathrm{CO}_{2}$. The realized $\mathrm{AVH}$ effect hosts the stacking pattern depended character. In addition, the AVH effect in $\mathrm{MnPSe}_{3} / \mathrm{SC}_{2} \mathrm{CO}_{2}$ can be switched on or off under ferroelectric switching of monolayer $\mathrm{Sc}_{2} \mathrm{CO}_{2}$.

\section{METHODS}

\section{Density functional theory calculations}

Our first-principles calculations are performed based on density functional theory (DFT) methods as implemented in the Vienna ab initio simulation package (VASP) ${ }^{62,63}$. The generalized gradient approximation (GGA) in form of Predew-Burke-Ernzerhof (PBE) functional is used to describe the exchange-correlation interaction ${ }^{64}$. The cutoff energy is set to $500 \mathrm{eV}$. Crystal structures are fully relaxed with the convergence criteria of $10^{-5} \mathrm{eV}$ and 0.01 eV $\AA^{-1}$ for energy and force, respectively. The Brillouin zone is sampled with Monkhorst-Pack grids of $9 \times 9 \times 1$. To avoid interactions between adjacent periodical structures, the vacuum space along the $z$ direction is set to $30 \AA$. The zero damping DFT-D3 method is utilized to treat the vdW interaction ${ }^{65}$. To describe the strong correlation effects, the effective on-site Hubbard term of $\mathrm{U}=4 \mathrm{eV}$ is set for the $3 \mathrm{~d}$ electrons of the $\mathrm{Mn}$ atom, as this value is employed in previous works ${ }^{44,66,67}$. Berry curvature is calculated using the maximally localized Wannier function method as implemented in the WANNIER90 package ${ }^{68}$. 


\section{DATA AVAILABILITY}

The authors declare that the data supporting the findings of this study are available within the paper and its supplementary information files.

\section{CODE AVAILABILITY}

The central codes used in this paper are VASP and WANNIER90. Detailed information related to the license and user guide are available at http://www.wannier.org and https://www.vasp.at.

Received: 1 September 2021; Accepted: 20 January 2022; Published online: 25 February 2022

\section{REFERENCES}

1. Xiao, D., Yao, W. \& Niu, Q. Valley-contrasting physics in graphene: magnetic moment and topological transport. Phys. Rev. Lett. 99, 236809 (2007).

2. Yao, W., Xiao, D. \& Niu, Q. Valley-dependent optoelectronics from inversion symmetry breaking. Phys. Rev. B 77, 235406 (2008).

3. Berkelbach, T., Hybertsen, M. \& Reichman, D. Theory of neutral and charged excitons in monolayer transition metal dichalcogenides. Phys. Rev. B 88, 045318 (2013).

4. Lu, H., Yao, W., Xiao, D. \& Shen, S. Intervalley scattering and localization behaviors of spin-valley coupled dirac fermions. Phys. Rev. Lett. 110, 016806 (2013).

5. Sanchez, O., Ovchinnikov, D., Misra, S., Allain, A. \& Kis, A. Valley polarization by spin injection in a light-emitting van der Waals heterojunction. Nano Lett. 16, 5792 (2016).

6. Schaibley, J. R. et al. Valleytronics in 2D materials. Nat. Rev. Mater. 1, 16055 (2016).

7. Vitale, S. A. et al. Valleytronics: opportunities, challenges, and paths forward. Small 14, 1801483 (2018).

8. Sham, L. J., Allen, S. J. Jr., Kamgar, A. \& Tsui, D. C. Valley-valley splitting in inversion layers on a high-index surface of silicon. Phys. Rev. Lett. 40, 472 (1978).

9. Ohkawa, F. J. \& Uemura, Y. Theory of valley splitting in an N-channel (100) inversion layer of Si I. Formulation by extended zone effective mass theory. $J$. Phys. Soc. Jpn 43, 907 (1977).

10. Rycerz, A., Tworzydlo, J. \& Beenakker, C. W. J. Valley filter and valley valve in graphene. Nat. Phys. 3, 172 (2007).

11. Akhmerov, A. R. \& Beenakker, C. W. J. Detection of valley polarization in graphene by a superconducting contact. Phys. Rev. Lett. 98, 157003 (2007).

12. $\mathrm{Wu}, \mathrm{S}$. et al. Electrical tuning of valley magnetic moment through symmetry control in bilayer $\mathrm{MoS}_{2}$. Nat. Phys. 9, 149 (2013).

13. Sie, E. J. et al. Valley-selective optical Stark effect in monolayerWS 2 . Nat. Mater. 14, 290 (2015).

14. Zhu, Z. Y., Cheng, Y. C. \& Schwingenschlögl, U. Giant spin-orbit-induced spin splitting in two-dimensional transition-metal dichalcogenide semiconductors. Phys. Rev. B 84, 153402 (2011).

15. Xiao, D., Liu, G.-B., Feng, W., Xu, X. \& Yao, W. Coupled spin and valley physics in monolayers of $\mathrm{MoS}_{2}$ and other Group-VI dichalcogenides. Phys. Rev. Lett. 108, 196802 (2012)

16. Li, X., Cao, T., Niu, Q., Shi, J. \& Feng, J. Coupling the valley degree of freedom to antiferromagnetic order. Proc. Natl Acad. Sci. USA 110, 3738 (2013).

17. Tong, W.-Y. \& Duan, C.-G. Electrical control of the anomalous valley Hall effect in antiferrovalley bilayers. npj Quant. Mater. 2, 47 (2017).

18. $\mathrm{Xu}$, L. et al. Large valley splitting in monolayer $\mathrm{WS}_{2}$ by proximity coupling to an insulating antiferromagnetic substrate. Phys. Rev. B 97, 041405 (2018).

19. $\mathrm{Ma}$, Y. et al. Conduction-band valley spin splitting in single-layer $\mathrm{H}-\mathrm{Tl}_{2} \mathrm{O}$. Phys. Rev. B 97, 035444 (2018)

20. Ke, C. et al. Large and controllable spin-valley splitting in two-dimensional $\mathrm{WS}_{2} / \mathrm{h}$ VN heterostructure. Phys. Rev. B 100, 195435 (2019).

21. Hu, H., Tong, W.-Y., Shen, Y.-H. \& Duan, C.-G. Electrical control of the valley degree of freedom in $2 \mathrm{D}$ ferroelectric/antiferromagnetic heterostructures. J. Mater. Chem. C. 8, 8098 (2020).

22. $\mathrm{Ma}, \mathrm{X}$. et al. Tunable valley splitting and anomalous valley Hall effect in $\mathrm{VTe}_{2} /$ $\mathrm{Ga}_{2} \mathrm{~S}_{3}$ heterostructures. J. Mater. Chem. C. 8, 14895 (2020).

23. Li, S. et al. Valley-dependent properties of monolayer $\mathrm{MoSi}_{2} \mathrm{~N}_{4}, \mathrm{WSi}_{2} \mathrm{~N}_{4}$, and $\mathrm{MoSi}_{2} \mathrm{As}_{4}$. Phys. Rev. B 102, 235435 (2020).

24. Yang, C., Song, Z., Sun, X. \& Lu, J. Valley pseudospin in monolayer $\mathrm{MoSi}_{2} \mathrm{~N}_{4}$ and $\mathrm{MoSi}_{2} \mathrm{As}_{4}$. Phys. Rev. B 103, 035308 (2021).

25. Dou, $\mathrm{K}$. et al. Promising valleytronic materials with strong spin-valley coupling in two-dimensional $\mathrm{MN}_{2} \mathrm{X}_{2}$ (M = Mo, W; X=F, H). Appl. Phys. Lett. 117, 172405 (2020).

26. Lei, $C$. et al. Nonvolatile controlling valleytronics by ferroelectricity in $2 \mathrm{H}-\mathrm{VSe}_{2} /$ $\mathrm{Sc}_{2} \mathrm{CO}_{2}$ van der Waals heterostructure. J. Phys. Chem. C. 125, 2802 (2021).
27. Mak, K. F., He, K., Shan, J. \& Heinz, T. F. Control of valley polarization in monolayer $\mathrm{MoS}_{2}$ by optical helicity. Nat. Nanotech. 7, 494 (2012).

28. Zeng, H., Dai, J., Yao, W., Xiao, D. \& Cui, X. Valley polarization in $\mathrm{MoS}_{2}$ monolayers by optical pumping. Nat. Nanotech. 7, 490 (2012).

29. Tong, W.-Y., Gong, S.-J., Wan, X. \& Duan, C.-G. Concepts of ferrovalley material and anomalous valley Hall effect. Nat. Commun. 7, 13612 (2016).

30. Liu, J. et al. Intrinsic valley polarization of magnetic $\mathrm{VSe}_{2}$ monolayers. J. Phys. Condens. Matter 29, 255501 (2017).

31. Zhao, P. et al. Single-layer $\mathrm{LaBr}_{2}$ : two-dimensional valleytronic semiconductor with spontaneous spin and valley polarizations. Appl. Phys. Lett. 115, 261605 (2019).

32. Song, Z. et al. Spontaneous valley splitting and valley pseudospin field effect transistors of monolayer $\mathrm{VAgP}_{2} \mathrm{Se}_{6}$. Nanoscale 10, 13986 (2018).

33. Peng, R. et al. Intrinsic anomalous valley Hall effect in single-layer $\mathrm{Nb}_{3} \mathrm{I}_{8}$. Phys. Rev. B 102, 035412 (2020).

34. Du, W. et al. Prediction of single-layer $\mathrm{TiVI}_{6}$ as a promising two-dimensional valleytronic semiconductor with spontaneous valley polarization. J. Mater. Chem. C. 8, 13220 (2020).

35. Zang, Y. et al. Large valley-polarized state in single-layer $\mathrm{NbX}_{2}(X=\mathrm{S}$, Se): theoretical prediction. Nano Res. 14, 834 (2021).

36. Cheng, H.-X., Zhou, J., Ji, W., Zhang, Y.-N. \& Feng, Y.-P. Two-dimensional intrinsic ferrovalley $\mathrm{Gdl}_{2}$ with large valley polarization. Phys. Rev. B 103, 125121 (2021).

37. Peng, R., Ma, Y., Zhang, S., Huang, B. \& Dai, Y. Valley polarization in Janus singlelayer MoSSe via magnetic doping. J. Phys. Chem. Lett. 9, 3612 (2018).

38. $\mathrm{Xu}, \mathrm{X}$. et al. Nonmetal-atom-doping-induced valley polarization in single-layer $\mathrm{Tl}_{2}$ O. J. Phys. Chem. Lett. 10, 4535 (2019).

39. Zhang, T. et al. Two-dimensional valleytronics in single-layer t-ZrNY $(\mathrm{Y}=\mathrm{Cl}, \mathrm{Br})$ predicted from first principles. J. Phys. Chem. C. 124, 20598 (2020).

40. Lei, $C$. et al. Valley polarization in monolayer $\mathrm{CrX}_{2}(\mathrm{X}=\mathrm{S}$, Se) with magnetically doping and proximity coupling. N. J. Phys. 22, 033002 (2020).

41. Aivazian, G. et al. Magnetic control of valley pseudospin in monolayer WSe $\mathrm{Wat}_{2}$. Phys. 11, 148 (2015).

42. Zhang, X.-X. et al. Magnetic brightening and control of dark excitons in monolayer WSe $\mathrm{W}_{2}$. Nat. Nanotech. 12, 883 (2017).

43. Qi, J., Li, X., Niu, Q. \& Feng, J. Giant and tunable valley degeneracy splitting in $\mathrm{MoTe}_{2}$. Phys. Rev. B 92, 121403 (2015).

44. Zhou, B., Li, Z., Wang, J., Niu, X. \& Luan, C. Tunable valley splitting and an anomalous valley Hall effect in hole-doped $\mathrm{WS}_{2}$ by proximity coupling with a ferromagnetic $\mathrm{MnO}_{2}$ monolayer. Nanoscale 11, 13567 (2019).

45. Pei, Q., Zhou, B., Mi, W. \& Cheng, Y. Triferroic material and electrical control of valley degree of freedom. ACS Appl. Mater. Interfaces 11, 12675 (2019).

46. Zhang, Z., Ni, X., Huang, H., Hu, L. \& Liu, F. Valley splitting in the van der Waals heterostructure $\mathrm{WSe}_{2} / \mathrm{Crl}_{3}$ : the role of atom superposition. Phys. Rev. B 99, 115441 (2019).

47. Jungwirth, T. et al. The multiple directions of antiferromagnetic spintronics. Nat. Phys. 14, 200 (2018).

48. Baltz, V. et al. Antiferromagnetic spintronics. Rev. Mod. Phys. 90, 015005 (2018)

49. Liu, G.-B., Shan, W.-Y., Yao, Y., Yao, W. \& Xiao, D. Three-band tight-binding model for monolayers of group-VIB transition metal dichalcogenides. Phys. Rev. B 88, 085433 (2013).

50. Chandrasekaran, A., Mishra, A. \& Singh, A. K. Ferroelectricity, antiferroelectricity, and ultrathin $2 \mathrm{D}$ electron/hole gas in multifunctional monolayer MXene. Nano Lett. 17, 3290 (2017).

51. Lee, C., Wei, X. D., Kysar, J. W. \& Hone, J. Measurement of the elastic properties and intrinsic strength of monolayer graphene. Science 321, 385 (2008).

52. Castellanos-Gomez, A. et al. Mechanical properties of freely suspended semiconducting graphene-like layers based on $\mathrm{MoS}_{2}$. Nanoscale Res Lett. 7, 233 (2012).

53. Halbertal, D. et al. Moiré metrology of energy landscapes in van der Waals heterostructures. Nat. Commun. 12, 242 (2021).

54. Li, L. \& Wu, M. Binary compound bilayer and multilayer with vertical polarizations: two-dimensional ferroelectrics, multiferroics, and nanogenerators. ACS Nano 11, 6382 (2017).

55. Liang, Y., Shen, S., Huang, B., Dai, Y. \& Ma, Y. Intercorrelated ferroelectrics in 2D van der Waals materials. Mater. Horiz. 8, 1683 (2021).

56. He, J., Li, S., Bandyopadhyay, A. \& Frauenheim, T. Unravelling photoinduced interlayer spin transfer dynamics in two-dimensional nonmagnetic-ferromagnetic van der Waals heterostructures. Nano Lett. 21, 3237 (2021).

57. Li, T. et al. Pressure-controlled interlayer magnetism in atomically thin $\mathrm{Crl}_{3}$. Nat. Mater. 18, 1303 (2019)

58. Yasuda, K., Wang, X., Watanabe, K., Taniguchi, T. \& Jarillo-Herrero, P. Stackingengineered ferroelectricity in bilayer boron nitride. Science 372, 1458 (2021).

59. Finney, N. R. et al. Tunable crystal symmetry in graphene-boron nitride heterostructures with coexisting moiré superlattices. Nat. Nanotechnol. 14, 1029 (2019).

60. Li, E. et al. Lattice reconstruction induced multiple ultra-flat bands in twisted bilayer $\mathrm{WSe}_{2}$. Nat. Commun. 12, 5601 (2021). 
61. Thouless, D. J., Kohmoto, M., Nightingale, M. P. \& den Nijs, M. Quantized Hall conductance in a two-dimensional periodic potential. Phys. Rev. Lett. 49, 405 (1982).

62. Kohn, W. \& Sham, L. J. Self-consistent equations including exchange and correlation effects. Phys. Rev. 140, A1133 (1965).

63. Kresse, G. \& Furthmüller, J. Efficient iterative schemes for ab initio total-energy calculations using a plane-wave basis set. Phys. Rev. B 54, 11169 (1996).

64. Perdew, J. P., Burke, K. \& Ernzerhof, M. Generalized gradient approximation made simple. Phys. Rev. Lett. 77, 3865 (1996).

65. Grimmea, S., Antony, J., Ehrlich, S. \& Krieg, H. A consistent and accurate ab initio parametrization of density functional dispersion correction (DFT-D) for the 94 elements H-Pu. J. Chem. Phys. 132, 154104 (2010).

66. Xue, F., Wang, Z., Hou, Y., Gu, L. \& Wu, R. Control of magnetic properties of $\mathrm{MnBi}_{2} \mathrm{Te}_{4}$ using a van der Waals ferroelectric $\mathrm{III}_{2}-\mathrm{Vl}_{3}$ film and biaxial strain. Phys. Rev. B 101, 184426 (2020).

67. Ma, X.-Y. et al. Large family of two-dimensional ferroelectric metals discovered via machine learning. Sci. Bull. 66, 233 (2021).

68. Mostofi, A. A. et al. An updated version of wannier90: a tool for obtaining maximally-localised Wannier functions. Comput. Phys. Commun. 185, 2309 (2014).

\section{ACKNOWLEDGEMENTS}

This work is supported by the National Natural Science Foundation of China (Nos 11804190 and 12074217), Shandong Provincial Natural Science Foundation (Nos. ZR2019QA011 and ZR2019MEM013), Shandong Provincial Key Research and Development Program (Major Scientific and Technological Innovation Project) (No. 2019JZZY010302), Shandong Provincial Key Research and Development Program (No. 2019RKE27004), Shandong Provincial Science Foundation for Excellent Young Scholars (No. ZR2020YQ04), Qilu Young Scholar Program of Shandong University, and Taishan Scholar Program of Shandong Province.

\section{AUTHOR CONTRIBUTIONS}

W.D. executed calculations. W.D., R.P. and Z.H. performed data analysis. Y.M. supervised the project. W.D. and Y.M. co-wrote the paper. All authors discussed the results and commented on the manuscript at all stages.

\section{COMPETING INTERESTS}

The authors declare no competing interests.

\section{ADDITIONAL INFORMATION}

Supplementary information The online version contains supplementary material available at https://doi.org/10.1038/s41699-022-00289-6.

Correspondence and requests for materials should be addressed to Ying Dai or Yandong Ma.

Reprints and permission information is available at http://www.nature.com/ reprints

Publisher's note Springer Nature remains neutral with regard to jurisdictional claims in published maps and institutional affiliations.
Open Access This article is licensed under a Creative Commons Attribution 4.0 International License, which permits use, sharing, adaptation, distribution and reproduction in any medium or format, as long as you give appropriate credit to the original author(s) and the source, provide a link to the Creative Commons license, and indicate if changes were made. The images or other third party material in this article are included in the article's Creative Commons license, unless indicated otherwise in a credit line to the material. If material is not included in the article's Creative Commons license and your intended use is not permitted by statutory regulation or exceeds the permitted use, you will need to obtain permission directly from the copyright holder. To view a copy of this license, visit http://creativecommons. org/licenses/by/4.0/.

(c) The Author(s) 2022 